\title{
Plasma properties in eruptive prominences
}

\author{
Nicolas Labrosse \\ SUPA, School of Physics and Astronomy, University of Glasgow, Glasgow G12 8QQ, UK \\ email: Nicolas.Labrosse@glasgow.ac.uk
}

\begin{abstract}
Prominence eruptions are one of the most spectacular manifestations of our Sun's activity. Yet there is still some mystery surrounding their relevant physical conditions. What are their plasma parameters? How different are they from those of quiescent prominences? How do they relate to those within coronal mass ejections? We briefly review some recent results in nonLTE radiative transfer modelling which contribute to our knowledge of the plasma properties in eruptive prominences. We discuss in particular how these results, combined with observational data analysis, can help us in determining the plasma parameters in eruptive prominences.
\end{abstract}

Keywords. Sun: activity, Sun: prominences

\section{Introduction}

Recent efforts in non-LTE (i.e. out of local thermodynamic equilibrium) modelling have been focused towards understanding how the prominence plasma evolves during an eruption. This is an important question to answer as it can shed light on CME initiation mechanisms, and on the link between the chromosphere and the heliosphere.

In this paper we first describe the effect of plasma motions on the emergent radiation, and detail the main aspects of the non-LTE modelling we used. Section 3 presents observations in the He II $304 \AA$ channel of SDO/AIA. Section 4 shows how the modelling results compare with these observations, and the ensuing diagnostic. Finally, we give conclusions in Section 5.

\section{Effects of plasma motions on emitted radiation}

Theoretical calculations have shown that when solar prominences move away from the surface of the Sun, their radiative output is affected via the Doppler dimming or brightening effects (Hyder \& Lites 1970; Heinzel \& Rompolt 1987; Gontikakis et al. 1997; Labrosse et al. 2007, 2008).

Doppler dimming (brightening) is the decrease (increase) in intensity of a line formed by resonant scattering of the incident radiation from the Sun due to the motion of the plasma. This effect is most commonly seen in strong resonance lines, and is widely used to diagnose the solar wind speed (Kohl \& Withbroe 1982).

In order to study the effects of radial motions on the spectral lines emitted by moving or erupting prominences, we adopt the simple case of a 1D plane-parallel vertical slab standing above the solar surface (Fig. 1). The code used is the same as described by Labrosse et al. (2007, 2008). The following equations are solved: hydrostatic equilibrium, ionisation and statistical equilibrium, and radiative transfer for Hydrogen and Helium. The prominence atmosphere model is defined by the choice of the gas pressure, temperature, column mass along the line-of-sight, altitude above the limb, and the radial velocity. The latter is used to define velocity-dependent boundary conditions for the radiative transfer equation (Heinzel \& Rompolt 1987). Pressure and temperature variations 


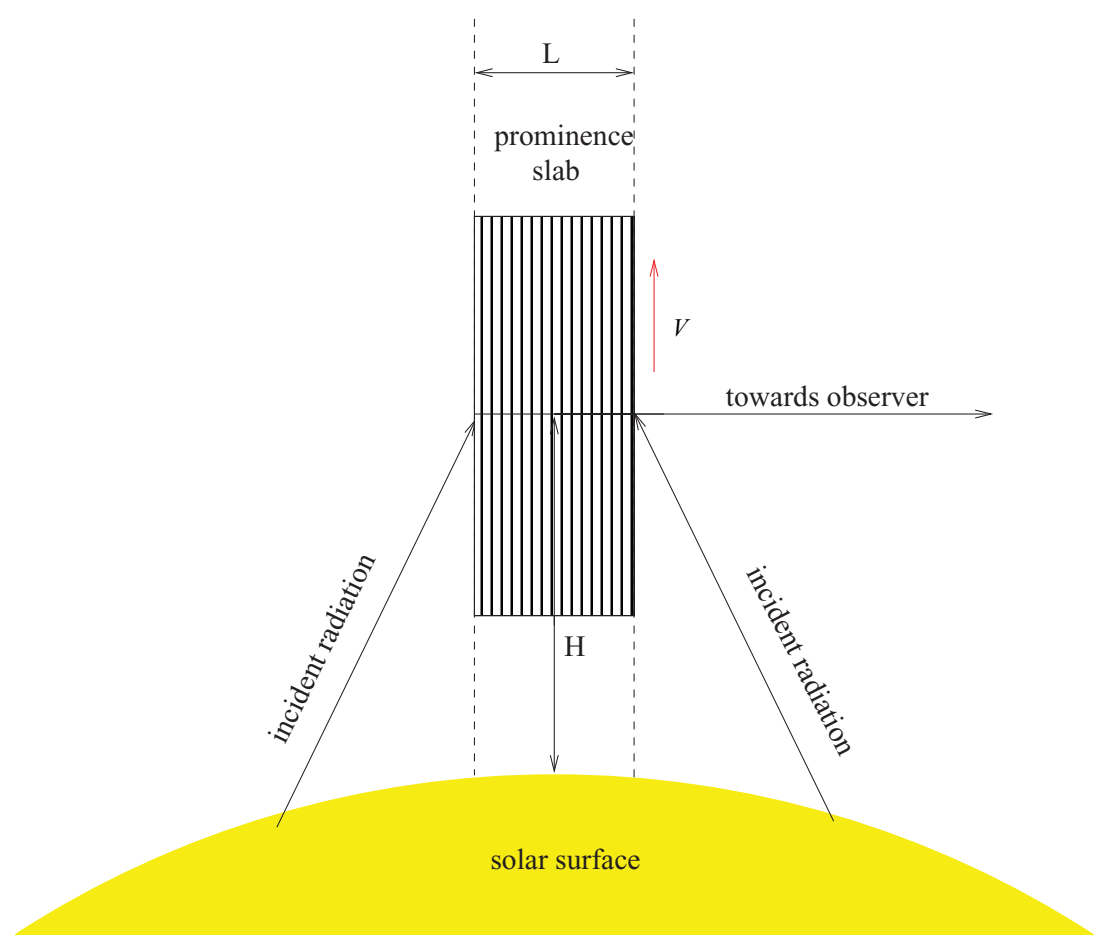

Figure 1. Sketch of the 1D plane-parallel prominence slab of geometrical width $L$, altitude $H$ above the solar surface, moving radially at a velocity $V$.

in the slab are described in Labrosse et al. (2008) and were taken from Anzer \& Heinzel (1999).

Similar multi-level, multi-line non-LTE modelling set-ups have been used by various authors to study radial motions in moving or erupting prominences, and the results are summarised in Labrosse et al. (2010). In short, the characteristics of the Doppler dimming or brightening effects on lines emitted by prominence plasma moving in the radial direction depend on:

- the line formation mechanisms, namely the relative importance of the resonant scattering mechanism with respect to other mechanisms such as collisional excitation

- the shape of the incident radiation, i.e. whether the incident line is in emission or in absorption, and its strength

- any coupling with other energy states or with other lines arising from statistical equilibrium and radiation transfer (see e.g. the case of the $\mathrm{H} \alpha$ line, well explained by Heinzel \& Rompolt 1987; Gontikakis et al. 1997).

Emergent line profiles are affected by the Doppler dimming or brightening effects to various degrees as per the above criteria. In the case of a strongly sensitive line affected by partial redistribution in frequency, asymmetries will arise between the red and the blue wings of the profile (Gontikakis et al. 1997).

It is worth noting that the relative importance of the resonant scattering mechanism, with respect to other excitation mechanisms, will depend on the actual radial velocity and plasma parameters. For example, when a significant amount of hot material is present in the prominence-to-corona transition region (hereafter PCTR), collisional processes may become more important in the line formation mechanisms, and compete with the resonant scattering mechanism. 


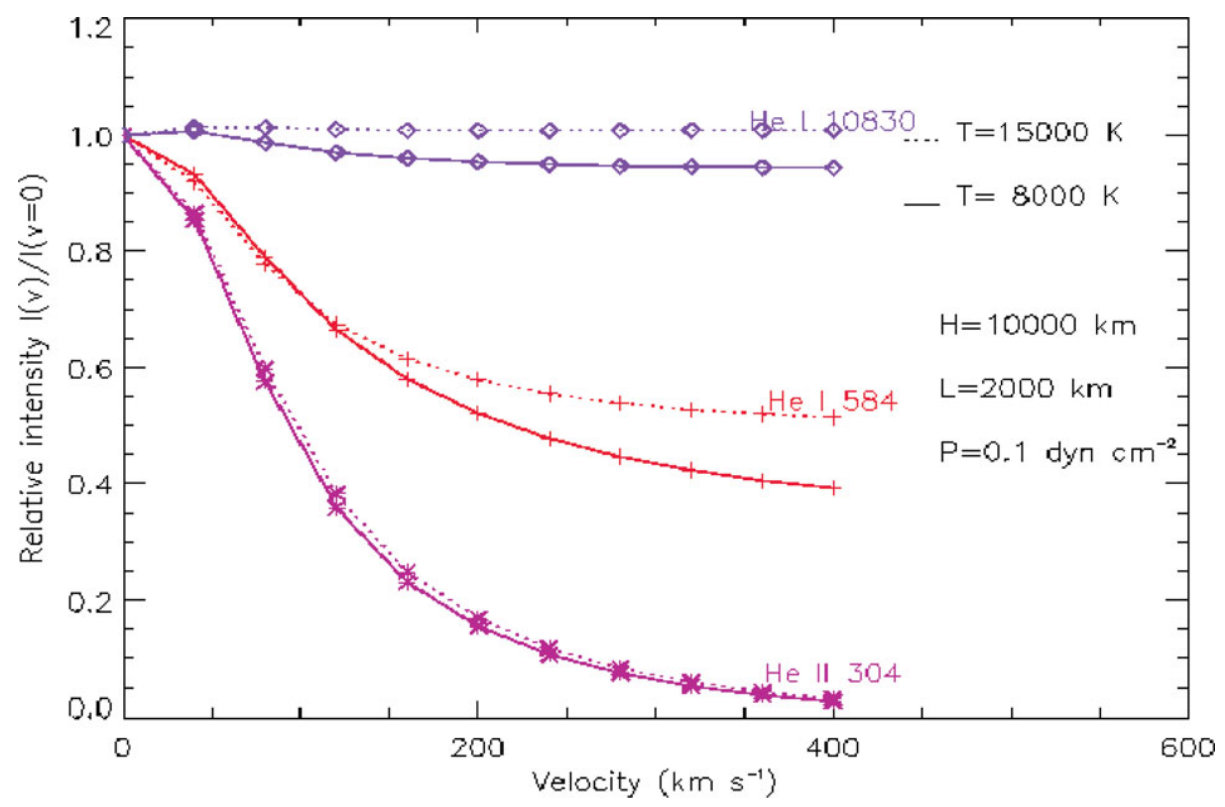

Figure 2. Relative intensity as a function of velocity at $8000 \mathrm{~K}$ (solid line) and $15000 \mathrm{~K}$ (dotted

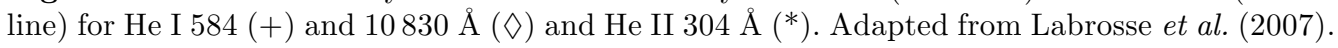

The investigations performed by Labrosse et al. $(2007,2008)$ showed that the He II $304 \AA$ line is very sensitive to the Doppler dimming effect. Under the prominence conditions considered in those studies, the main formation mechanism of that line is the scattering of the strong He II $304 \AA$ emission line coming from the disk (Fig. 2). Hence it was shown that the Doppler dimming effect on the He II 304 line is stronger when most of the prominence plasma is cool, not too dense, or a large temperature gradient exists in the PCTR. When the physical conditions deviate from this situation, the relative contribution from the thermal processes becomes more and more important in the formation of the line - the relative contribution of the resonant scattering becomes smaller and smaller - and the sensitivity to the Doppler dimming effect is weaker.

It is important to stress that in all the previously referenced studies, the modelled prominence plasma parameters are kept constant while the radial velocity is changed in order to investigate the effects of radial motions on the radiation emitted by eruptive prominences.

\section{Observations}

Taking the theoretical results discussed in the previous section, the natural question to ask is then: Is Doppler dimming observed in eruptive prominences? A first step in answering this question was taken in Labrosse \& McGlinchey (2012). In that paper, the authors asked whether observational signatures of the changes in the radiative output of eruptive prominences could be found in EUV observations of the He II $304 \AA$ line. The authors studied the variation of intensity of arbitrarily selected features in the 304 channel from SDO/AIA observations of prominence eruptions as a function of velocity in the plane of the sky. These results were then compared with non-LTE radiative transfer calculations of the intensity of the He II 304 resonance line.

It was found that the observed intensity variations in various parts of the four eruptive prominences studied were not always consistent with the Doppler dimming effect on the 


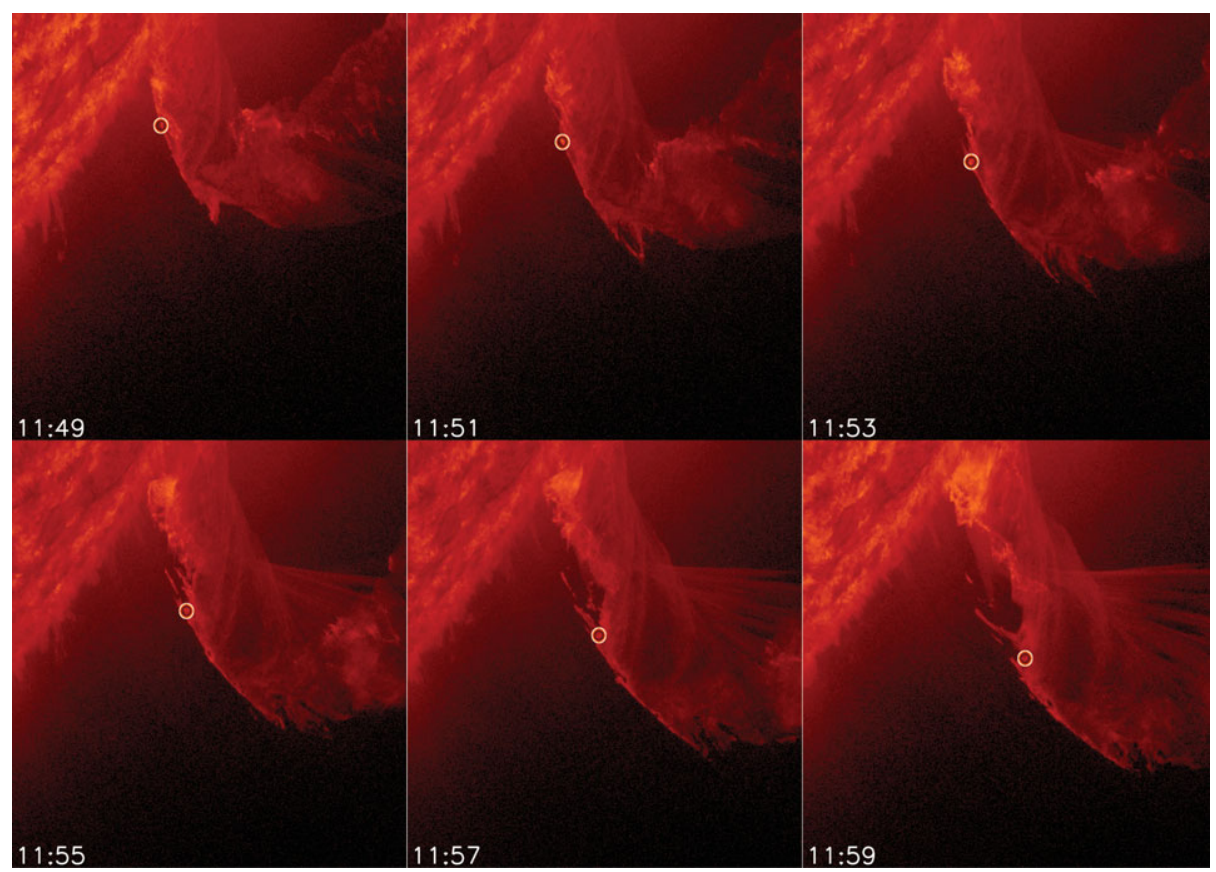

Figure 3. Evolution of the 2011-03-19 prominence eruption. The white circle marks the part of the prominence which was tracked and used to calculate the intensities as a function of altitude and velocity. The field of view is $390^{\prime \prime} \times 390^{\prime \prime}$.

He II $304 \AA$ line presented thus far. Namely, in some cases one observes an increase in intensity in the 304 channel with velocity, in contradiction to what is expected from the Doppler dimming effect alone. The use of new non-LTE models allowed the authors to explain the different behaviour of the intensity by changes in the plasma parameters inside the prominence, in particular the column mass of the plasma. The non-LTE models used in that study were more realistic than those used in previous calculations and discussed in the previous section. The main improvement is that they took into consideration the variation of the plasma parameters during the eruption (as opposed to keeping the plasma parameters constant, as in Fig. 2).

\section{Prominence plasma diagnostic}

We can now investigate whether these observations can be used to perform a diagnostic of the plasma of the eruptive prominence. We focus on one of the events presented in Labrosse \& McGlinchey (2012) - the 19 March 2011 eruption observed by SDO/AIA (Fig. 3). We follow the same method as in Labrosse \& McGlinchey (2012), although we have included some improvements in the comparison between observations and simulations.

\subsection{Normalisation of the model intensities}

The observed intensity data from AIA is normalised to the intensity that corresponds to the lowest observed radial velocity. In Labrosse \& McGlinchey (2012), a model with zero radial velocity was designated as the reference model, and the modelled intensities were normalised to this reference model. This led to a radial velocity difference between the lowest observed and the reference model radial velocity. Additionally, the normalisation 


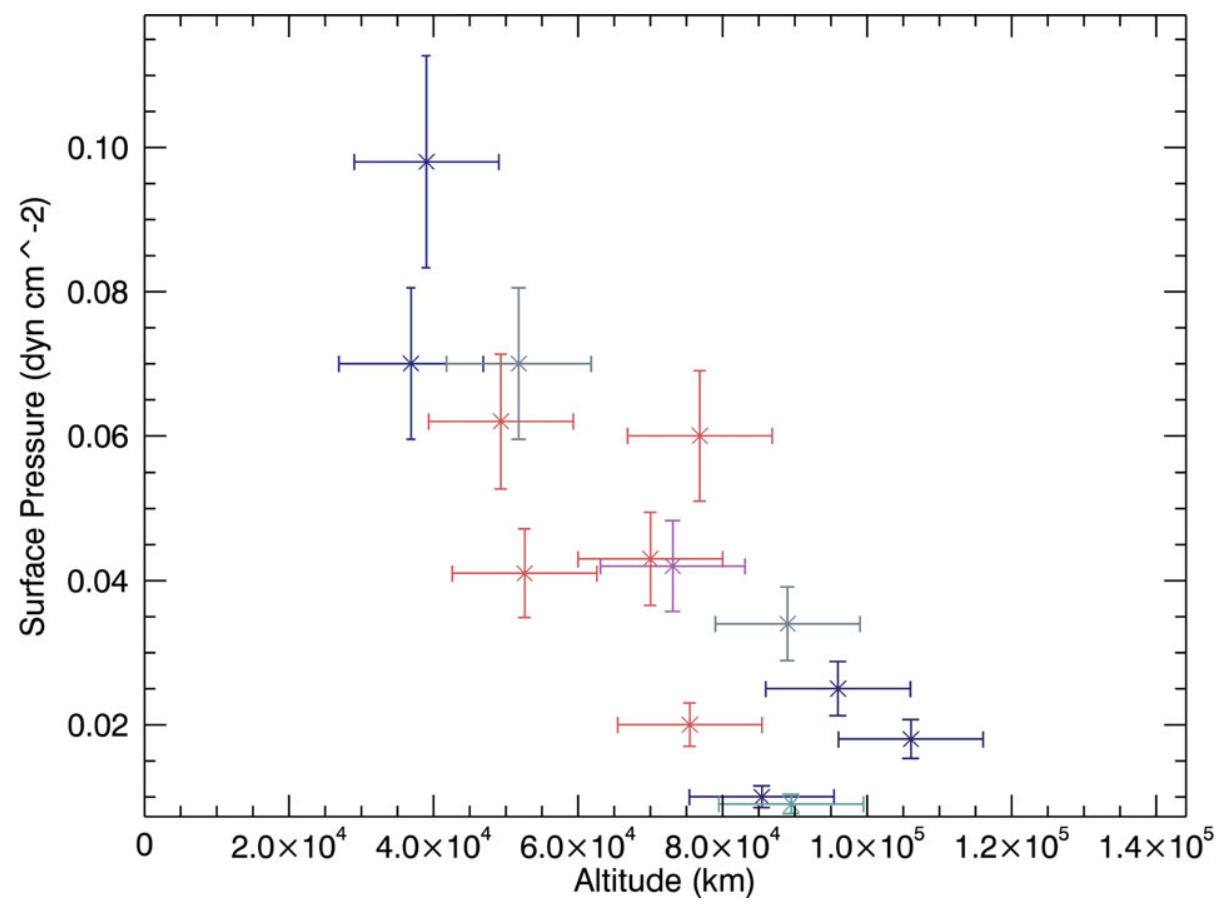

Figure 4. Surface pressure as a function of altitude for the selected models matching observations of the 19 March 2011 eruption.

did not account for the observed altitude, which led to differences in observed and reference model altitudes.

We improve upon this technique by creating a set of models with radial velocities fixed to the value of the lowest observed radial velocity. This results in multiple appropriate radial velocity models in which we can search for the model with the closest altitude to the observed altitude. This model is then designated as the reference model corresponding to the chosen observation data set.

This new normalisation procedure is far closer to the normalisation undergone by the observed intensities. This allows for accurate comparison between observation data and model data, an essential step in the prominence diagnostic process.

\subsection{Model search}

Each model is defined by the three observed parameters (intensities, radial velocities, and altitudes), along with other prominence plasma parameters. It is therefore possible to search the models for those which are probable matches for each observation point. We take each observation point and search for models which have altitude, intensity at $304 \AA$ and radial velocity within the error boundaries of those observed parameters. Acceptable models are then combined across the lifetime of the prominence, and analysed.

\subsection{Results}

Figure 4 shows the results of this diagnostic method. It suggests that the surface pressure in the erupting prominence decreases as the eruption unfolds. This is consistent with the decrease in pressure with altitude in the corona. 


\section{Conclusions}

We have shown here that we can obtain plasma parameters in eruptive prominences by comparing and minimising the differences between observations and non-LTE model results. As an example, we observe the decrease of the pressure at the surface of an eruptive prominence as its altitude in the corona increases, going from $0.1 \mathrm{dyn} \mathrm{cm}^{-2}$ at

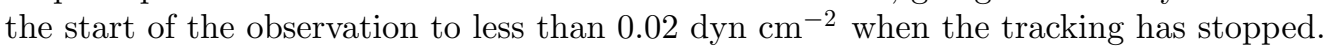

This technique can be applied to any instrument that will monitor prominence eruptions in a resonantly scattered line such as Hydrogen and He II Lyman lines. In the future, the EUI instrument on Solar Orbiter will monitor the low atmosphere counterparts of large-scale solar eruptive events such as CMEs (see Auchere's paper in this issue). It will be ideally suited to study prominence eruptions. Using both H Ly- $\alpha$ and He II 304 lines will add constraints on the models. In addition, combining EUI observations with in-situ data out of the ecliptic plane will bring invaluable insights on the physical conditions within EPs, and a better understanding of the links between eruptive prominences and the dynamic heliosphere.

Acknowledgement: The author acknowledges the help of Scott Forrest in this work.

\section{References}

Anzer, U. \& Heinzel, P. 1999, A\&A, 349, 974

Gontikakis, C., Vial, J.-C., \& Gouttebroze, P. 1997, A\&A, 325, 803

Heinzel, P. \& Rompolt, B. 1987, Sol. Phys., 110, 171

Hyder, C. L. \& Lites, B. W. 1970, Sol. Phys., 14, 147

Kohl, J. L. \& Withbroe, G. L. 1982, ApJ, 256, 263

Labrosse, N., Gouttebroze, P., \& Vial, J.-C. 2007, A\&A, 463, 1171

Labrosse, N., Heinzel, P., Vial, J., et al. 2010, Space Science Reviews, 151, 243

Labrosse, N. \& McGlinchey, K. 2012, A\&A, 537, A100

Labrosse, N., Vial, J.-C., \& Gouttebroze, P. 2008, Annales Geophysicae, 26, 2961 